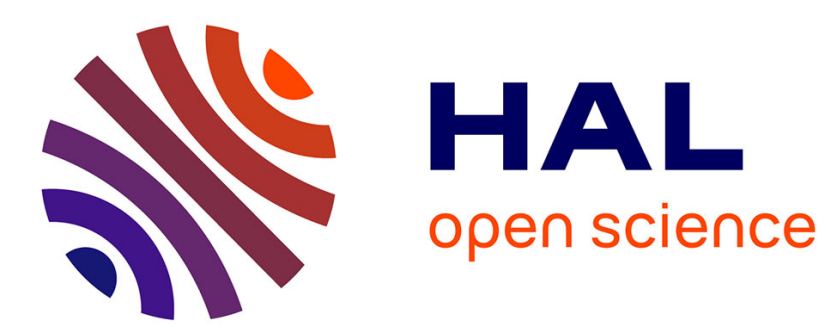

\title{
Structural studies of the CDW interaction with defects
} S. Ravy, Jean Pouget

\section{To cite this version:}

S. Ravy, Jean Pouget. Structural studies of the CDW interaction with defects. Journal de Physique IV Proceedings, 1993, 03 (C2), pp.C2-109-C2-114. 10.1051/jp4:1993221 . jpa-00251304

\section{HAL Id: jpa-00251304 https://hal.science/jpa-00251304}

Submitted on 1 Jan 1993

HAL is a multi-disciplinary open access archive for the deposit and dissemination of scientific research documents, whether they are published or not. The documents may come from teaching and research institutions in France or abroad, or from public or private research centers.
L'archive ouverte pluridisciplinaire HAL, est destinée au dépôt et à la diffusion de documents scientifiques de niveau recherche, publiés ou non, émanant des établissements d'enseignement et de recherche français ou étrangers, des laboratoires publics ou privés. 


\title{
Structural studies of the CDW interaction with defects
}

\author{
S. RAVY and J.P. POUGET
}

Laboratoire de Physique des Solides, URA CNRS 02, bât. 510, Université Paris-Sud, 91405 Orsay, France

\begin{abstract}
We present new $X$-ray diffuse scattering effects due to pinning of the phase of charge density waves (CDW's) on defects. The coherence between the position of a defect and the phase of a $2 k_{F}$ periodic lattice distortion gives rise to an asymmetry of the $+2 k_{F} /-2 k_{F}$ diffuse intensity with respect to the layers of main Bragg reflections. This effect, negligible in the weak pinning limit, becomes dominant for strong pinning. In addition, CDW phase distortions around defects produce an asymmetry of each individual $2 \mathrm{k}_{\mathrm{F}}$ diffuse lines. We first calculate the scattered intensity in these different cases that we illustrate by experimental results taken from charge transfer organic salts of the TTF-TCNQ family.
\end{abstract}

\section{General formulation}

Let us consider a one-dimensional (1D) lattice of period a of $\mathrm{N}$ sites containing $\mathrm{A}$ impurities in concentration $x$ among $B$ species (atoms or molecules). We define the $\sigma_{n}$ variable by $\sigma_{n}=1$ if the site $n$ is occupied by the A specie and $\sigma_{n}=0$ otherwise. Let us now consider a lattice displacement $\mathbf{u}_{n}$ associated to a CDW of wave vector $\mathbf{q}$. The $\mathrm{X}$-ray scattered amplitude at the scattering vector $\mathbf{Q}=\mathbf{G}+\mathbf{q}$ is, in first order in $\left(\mathbf{Q} \cdot \mathbf{u}_{\mathbf{q}}\right)$, given by $[1]$

$$
\mathrm{A}(\mathbf{Q})=\Delta \mathbf{f} \sigma_{\mathbf{q}}+\mathrm{i} \mathbf{f}\left(\mathbf{Q} \cdot \mathbf{u}_{\mathbf{q}}\right),
$$

where $\mathbf{G}$ is a reciprocal wave vector, $\Delta f=\left(f_{A}-f_{B}\right)$ is the difference between the scattering factors of the $A$ and $B$ species, $f=x f_{A}+(1-x) f_{B}$ is the average scattering factor and $\sigma_{q}$ and $\mathbf{u}_{\mathbf{q}}$ are the Fourier components of the $\sigma_{\mathrm{n}}$ and $\mathbf{u}_{\mathrm{n}}$ functions defined by

$$
\mathbf{u}_{\mathbf{q}}=\frac{1}{\mathrm{~N}} \sum_{\mathrm{n}} \mathbf{u}_{\mathrm{n}} \mathrm{e}^{\mathrm{iqna}} \text { and } \sigma_{\mathbf{q}}=\frac{1}{\mathrm{~N}} \sum_{\mathrm{n}} \sigma_{\mathrm{n}} \mathrm{e}^{\mathrm{iqna}}
$$

From equation (1) it follows that the scattered intensity is the sum of three terms: 
- disord. $_{\text {dister }}=|\Delta \mathrm{f}|^{2}\left|\sigma_{\mathbf{q}}\right|^{2}$, which is the diffuse scattering intensity due to disorder only. In the case of a random disorder this scattering is called Laue scattering and $I_{\text {Laue }}=x(1-x)\left|f_{A}-f_{B}\right|^{2}$.

$-I_{\text {disp. }}=f^{2}\left|\mathbf{Q} \cdot \mathbf{u}_{\mathbf{q}}\right|^{2}$, which is the intensity due to the displacive modulation $\mathbf{u}_{\mathbf{n}}$. In our $1 D$ example, this scattering gives rise to diffuse lines at $\pm q$ from the layers of Bragg reflections located at $\mathbf{G}$.

$-\mathrm{I}_{\mathbf{A}}=\mathrm{i}$ f $\Delta \mathrm{f}\left(\sigma_{\mathbf{q}}{ }^{*}\left(\mathbf{Q} \cdot \mathbf{u}_{\mathbf{q}}\right)-\sigma_{\mathbf{q}}\left(\mathbf{Q} \cdot \mathbf{u}_{\mathbf{q}}\right)^{*}\right)$ which gives interferences between the disorder and the lattice displacements. As $\sigma_{n}$ and $u_{n}$ are real, we obtain $I_{A}(G+q)=-I_{A}(G-q)$ by using $\mathbf{u}_{-\mathbf{q}}=\mathbf{u}_{\mathbf{q}}{ }^{*}$ and $\sigma_{-\mathbf{q}}=\sigma_{\mathbf{q}}{ }^{*}$ and neglecting the $\mathbf{Q}$ dependence of the scattering factors. This term causes the $+q /-q$ asymmetry around the Bragg reflections layers. Moreover, as $I_{A}$ linearly depends on the displacement $\mathbf{u}_{\mathbf{q}}$ its contribution is expected to be larger than $I_{\text {disp. }}$, which has the usual quadratic $\mathbf{u}_{\mathbf{q}}$ dependence for small values of $\mathbf{Q} \cdot \mathbf{u}_{\mathbf{q}}$. As we shall see this term has a great importance in the case of a CDW pinning.

Let us now calculate $\mathrm{I}_{\mathrm{A}}$ for different $1 \mathrm{D}$ models of $\mathrm{CDW}$ pinning.

\section{Case of strong pinning}

Let us consider that each impurity induces a Friedel oscillation or pins the phase of the $\mathrm{CDW}$ with the same constant value. Around an impurity located at site $\mathrm{m}$ we will write the displacement $\mathbf{u}_{\mathrm{n}}^{\mathrm{m}}$ as

$$
\mathbf{u}_{\mathrm{n}}^{\mathrm{m}}=\sum_{\mathbf{q}} \mathbf{u}_{\mathbf{q}}^{\prime} \sin \left(\mathbf{q}(\mathrm{n}-\mathrm{m}) \mathbf{a}+\varphi_{0}\right)
$$

where $\mathbf{u}_{\mathbf{q}}^{\prime}$ is a function of $\mathbf{q}$ centred around $2 \mathbf{k}_{F}$ ( $\mathbf{k}_{F}$ being the Fermi wave vector) and $\varphi_{0}$ the phase of the distortion with respect to the impurity. $\dot{u}_{\mathbf{q}}^{\prime}$ is the Fourier transform of each individual distortion.

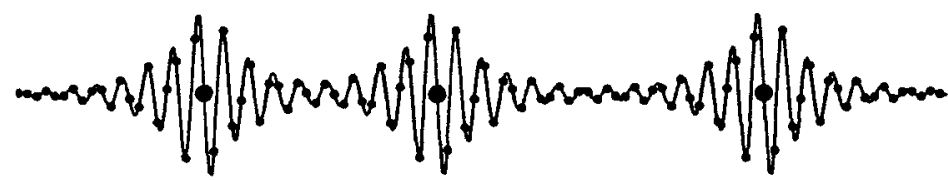

a)

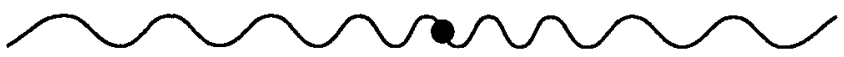

b)

Figure 1 : Schematic representation of lattice displacements in the case where each impurity a) pins the phase at a constant value and b) induces a local deformation of the phase of the modulation. 
If we assume that the total displacement at site na is the sum of the displacements around each impurity as schematically shown fig. 1 a) we obtain

$$
\mathbf{u}_{\mathrm{n}}=\sum_{\mathrm{m}} \sigma_{\mathrm{m}} \sum_{\mathbf{q}} \mathbf{u}_{\mathbf{q}} \sin \left(\mathbf{q}(\mathrm{n}-\mathrm{m}) \mathbf{a}+\varphi_{0}\right)
$$

This expression implicitely assumes a smooth variation of the phase between the defects in order to keep the same $\varphi_{0}$ on each impurity site. After some algebra one gets

$$
\mathbf{u}_{\mathbf{q}}=\frac{\mathrm{i}}{2} \mathrm{~N} \sigma_{\mathbf{q}} \dot{u}_{\mathbf{q}} \mathrm{e}^{-\mathrm{i} \varphi_{0}}
$$

With this value $\mathrm{I}_{\mathrm{A}}(\mathbf{G}+\mathbf{q})$ becomes :

$$
\mathrm{I}_{\mathbf{A}}(\mathbf{G}+\mathbf{q})=-\mathrm{N} \mathbf{f} \Delta \mathbf{f}\left(\mathbf{Q} \cdot \mathbf{u}_{\mathbf{q}}^{\prime}\right)\left|\sigma_{\mathbf{q}}\right|^{2} \cos \varphi_{0}
$$

From this equation it is clear that $\mathrm{I}_{A}$ shows an extremum when $\varphi_{0}=0$ or $\pi$. In these cases the asymmetry of the intensity of the $+2 \mathbf{k}_{\mathrm{F}} /-2 \mathbf{k}_{\mathrm{F}}$ diffuse lines will be maximum. More generally, it can be shown that this occurs when a displacement wave and a chemical wave are in quadrature [2], which is the case of a strong pinning of CDW's on a defect. As previously mentionned, this asymmetry should dominate $I_{\text {disp }}$ for small Q. $\mathbf{u}_{\mathbf{q}}$, which is the case for $1 D$ conductors such as TTF-TCNQ ( a typical value of $\mathbf{u}_{\mathbf{q}}{ }^{[3]}$ gives $\mathbf{Q} \cdot \mathbf{u}_{\mathbf{q}} \sim 2 \pi \AA-10.01 \AA \sim 0.06$ ).
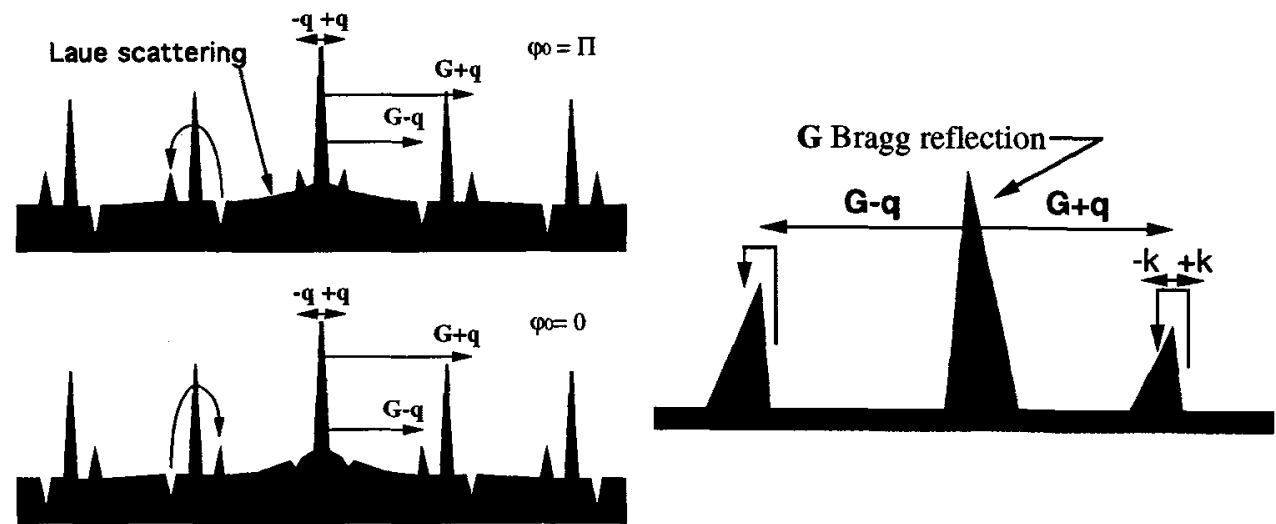

a)

b)

Figure 2 : Schematic representation of the intensity scattered by a modulated chain where each impurity a) pins a lattice modulation of wave vector $q$ with the phase $\varphi_{0}=\pi$ and 0 (top and bottom) and $b$ ) induces a phase distortion of the modulation.

As a result, when $I_{A}(Q)$ is negative, the diffuse lines will appear at the expense of the disorder induced Laue scattering background. As shown figure 2a), the position of these "white lines" allows to determine the phase of the lattice distortion with respect to the impurity ${ }^{[4]}$ provided the sign of $f . \Delta f .\left(Q^{\prime} \cdot \mathbf{u}_{\mathbf{q}}\right.$ ) (taken positive in fig. $2 \mathrm{a}$ ) ) is known.

\section{Case of weak pinning}

As previously shown the effect of $+2 k_{F} /-2 k_{F}$ asymmetry around $\mathbf{G}$ comes from the coherence between the position of a defect and the phase of the periodic lattice distortion. 
When the value of the phase $\varphi_{0}$ is not the same on every impurity site, as in the weak pinning limit considered by Fukuyama-Lee-Rice[11], we will have the impurity average $\left\langle\cos \varphi_{0}\right\rangle \cong 0$ for sufficiently large domains, so that the interference term $\mathrm{I}_{\mathrm{A}}$ will become negligible with respect to $\mathrm{I}_{\text {disp. }}$. No $+2 \mathbf{k}_{\mathrm{F}} /-2 \mathbf{k}_{\mathrm{F}}$ asymmetry is thus expected.

\section{Phase deformation around a defect.}

Let us now consider a deformation of the phase of the CDW around a defect as schematically represented figure $1 b)$. This local deformation can be written

$$
\mathbf{u}_{\mathrm{n}}^{\mathrm{m}}=\mathbf{u}_{2 \mathbf{k}_{\mathbf{F}}} \sin \left[2 \mathbf{k}_{\mathrm{F}}(\mathrm{n}-\mathrm{m}) \mathbf{a}+\varphi_{0}+\varphi(\mathrm{n}-\mathrm{m})\right] \text {. }
$$

Assuming that $\varphi(n-m)$ is an odd function (whose Fourier components are $\varphi_{\mathbf{k}}$ ) and a small quantity one gets

$$
\mathbf{u}_{\mathrm{n}}^{\mathrm{m}}=\mathbf{u}_{2 \mathbf{k}_{\mathrm{F}}} \sin \left[2 \mathbf{k}_{\mathbf{F}}(\mathrm{n}-\mathrm{m}) \mathbf{a}+\varphi_{0}\right]+\mathbf{u}_{2 \mathbf{k}_{\mathrm{F}}} \sum_{\mathbf{k}} \varphi_{\mathbf{k}} \sin (\mathbf{k}(\mathrm{n}-\mathrm{m}) \mathbf{a}) \cos \left[2 \mathbf{k}_{\mathbf{F}}(\mathrm{n}-\mathrm{m}) \mathbf{a}+\varphi_{0}\right]
$$

The first term of the right side gives rise to the $+2 \mathrm{k}_{\mathrm{F}} /-2 \mathrm{k}_{\mathrm{F}}$ asymmetry previously described by (2) and from the second term one obtains the following values of $\mathbf{u}_{\mathbf{q}=2 \mathbf{k r} \pm \mathbf{k}}$ :

$$
\mathbf{u}_{\mathbf{q}=2 \mathbf{k} F+\mathbf{k}}=\frac{\mathrm{i}}{4} \mathrm{~N} \sigma_{2 \mathbf{k} F+\mathbf{k}} \mathbf{u}_{2 \mathbf{k}_{\mathrm{F}}} \varphi_{\mathbf{k}} \mathrm{e}^{-\mathrm{i} \varphi_{0}} \text { and } \mathbf{u}_{\mathbf{q}=2 \mathbf{k} \boldsymbol{F}-\mathbf{k}}=-\frac{\mathrm{i}}{4} \mathrm{~N} \sigma_{2 \mathbf{k}_{\mathrm{F}-\mathbf{k}}} \mathbf{u}_{2 \mathbf{k}_{\mathrm{F}}} \boldsymbol{\varphi}_{\mathbf{k}} \mathrm{e}^{-\mathrm{i} \varphi_{0}}
$$

The substitution of these quantities in the expression of $I_{A}$ gives

$$
\begin{aligned}
& I_{A}\left(\mathbf{G}+2 \mathbf{k}_{F}+\mathbf{k}\right)=-\mathrm{N} / 2 \mathrm{f} . \Delta \mathbf{f}\left|\sigma_{2 \mathbf{k F}+\mathbf{k}}\right|^{2}\left(\mathbf{Q} \cdot \mathbf{u}_{2 \mathbf{k F}}\right) \varphi_{\mathbf{k}} \cos \varphi_{0} \text { and } \\
& \mathbf{I}_{\mathrm{A}}\left(\mathbf{G}+2 \mathbf{k}_{\mathrm{F}}-\mathbf{k}\right)=+\mathrm{N} / 2 \mathrm{f} . \Delta \mathbf{f}\left|\sigma_{2 \mathbf{k} F-\mathbf{k}}\right|^{2}\left(\mathbf{Q} \cdot \mathbf{u}_{2 \mathbf{k}}\right) \varphi_{\mathbf{k}} \cos \varphi_{0},
\end{aligned}
$$

The same relations are obtained for $\mathbf{G}-2 \mathbf{k}_{\mathrm{F}}+\mathbf{k}$ and $\mathbf{G}-2 \mathbf{k}_{\mathrm{F}}-\mathbf{k}$ respectively.

These equations show that in the case of an antisymmetric phase deformation around an impurity, the individual $\pm 2 \mathbf{k}_{\mathrm{F}}$ lines are themselves asymmetric as sketched figure $2 \mathrm{~b}$ ). This effect is analoguous to the so-called size effect[5] where Bragg reflections with asymmetric tails are observed due to the elastic deformations of the lattice around the defects. Let us note that the sense of the $+k /-k$ asymmetry of the $2 k_{F}$ diffuse lines allows to determine the sense of the phase distortion (i.e. the sign of $\varphi_{\mathbf{k}}$ ) provided that the signs of $f, \Delta f, \cos \varphi_{0}$ and $\mathbf{Q} \cdot \mathbf{u}_{\mathbf{2}}$ are known. However, as these terms are also present in the expression of $\mathrm{I}_{\mathrm{A}}$ given by equation (2), the simultaneous observation of the $+2 \mathbf{k}_{\mathrm{F}} /-2 \mathbf{k}_{\mathrm{F}}$ asymmetry around $\mathbf{G}$ and of a $+\mathbf{k} /-\mathbf{k}$ asymmetry of each $2 \mathbf{k}_{\mathrm{F}}$ diffuse line allows to determine the sign of $\varphi_{\mathbf{k}}$. It is found that $\varphi_{\mathbf{k}}>0$ $(<0)$ if both kinds of asymmetry occur in the same (opposite) direction.

\section{Experimental results}

All the effects previously described have been observed in 1D charge transfer salts of the TTF-TCNQ family. Figure 3a) shows an X-ray pattern obtained from the solid solution (HMTTF) 0.87 (HMTSF) 0.13 TCNQ. White diffuse lines at $\mathbf{G}+2 \mathbf{k}_{\mathrm{F}}$ and $\mathbf{G}+4 \mathbf{k}_{\mathrm{F}}$ are clearly 
visible. A more general study of the $(\mathrm{HMTTF})_{1-\mathrm{x}}(\mathrm{HMTSF})_{\mathrm{X}}$ TCNQ and $(\mathrm{TTF})_{1-\mathrm{x}}(\mathrm{TSF})_{\mathrm{X}}$ TCNQ solid solutions shows that :

-The white lines appears at

i) $\mathbf{G}+2 \mathbf{k}_{\mathbf{F}}$ in (HMTTF) 0.05 (HMTSF) $0.95 \mathrm{TCNQ}^{[4]}$,

ii) $\mathbf{G}+2 \mathbf{k}_{\mathrm{F}}$ and $\mathbf{G}+4 \mathbf{k}_{\mathrm{F}}$ in (HMTTF) 0.87 (HMTSF) 0.13 TCNQ (fig. 3a)

iii) $\mathbf{G}+4 \mathbf{k}_{\mathrm{F}}$ in $(\mathrm{TTF})_{0.97}(\mathrm{TSF})_{0.03} \mathrm{TCNQ}[10]$.

These results indicate that :

- the donor stacks bear the instability which shows the white line effect. In particular:

i) - the HMTSF chains exhibit the $2 \mathbf{k}_{\mathrm{F}}$ instability.

ii) - the HMTTF chains exhibit both $2 \mathbf{k}_{\mathrm{F}}$ and $4 \mathbf{k}_{\mathrm{F}}$ instabilities.

iii) - the TTF chains exhibit the $4 \mathbf{k}_{F}$ instability.

-the $2 \mathrm{k}_{\mathrm{F}}$ and/or $4 \mathrm{k}_{\mathrm{F}}$ lattice distortions are pinned to the $\mathrm{S}(\mathrm{Se})$ based molecular substituants with the phases $\varphi_{0}=\pi(0)$ which corresponds to a displacement of the first neighboring molecule towards (away from) the molecular substituant.

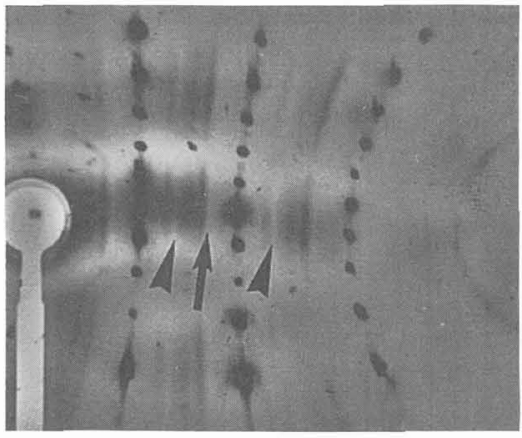

a)

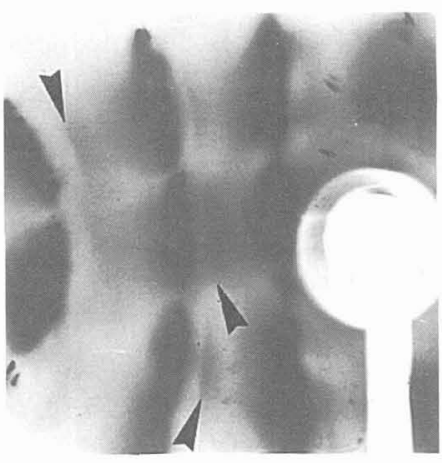

b)

Figure 3: a) X-ray pattern from the solid solution (HMTTF) 0.87 (HMTSF) 0.13 TCNQ at $20 \mathrm{~K}$ showing the $+2 \mathbf{k}_{\mathrm{F}}$ (short arrows) and $+4 \mathbf{k}_{\mathrm{F}}$ (long arrow) white diffuse lines $(\lambda=\mathrm{CuK} \alpha)$.

b) X-ray pattern from TMTSF-DMTCNQ with $2.8 \%$ irradiation defects at $10 \mathrm{~K}[6]$. Arrows show the asymmetric $2 \mathbf{k}_{\mathrm{F}}$ diffuse lines $(\lambda=\mathrm{CuK} \alpha)$. A schematic representation of the asymmetries of intensity of this X-ray pattern is given by fig. 2b).

Figure $3 b$ ) shows clearly the asymmetry of the $2 \mathbf{k}_{\mathrm{F}}=0.25 \mathbf{a}^{*}$ diffuse lines in the case of TMTSF-DMTCNQ containing $2.8 \%$ irradiation defects $[6]$. An asymmetry of the cloud of diffuse scattering around the $\mathrm{h}=$ constant layers of Bragg reflections is also visible. This latter effect is the signature of strong elastic distortions around defects[7]. According to the calculations of ref. [7], this asymmetry towards the large reciprocal wave vector values corresponds to a lattice dilatation in the vicinity of the defects, a result which agrees with the observation that lattice parameters increase in irradiated samples ${ }^{[8]}$. The asymmetry of the $2 \mathbf{k}_{\mathrm{F}}$ 
diffuse line indicates that there is a strong distortion of the phase of the CDW in the vicinity of the defects. Nevertheless, the absolute determination of the sign of this distortion requires a determination of the sign of $\Delta \mathrm{f}$ which is a complex function of the displacement field in the case of strongly distorded crystals [7]. However as a $+2 \mathbf{k}_{\mathrm{F}} /-2 \mathbf{k}_{\mathrm{F}}$ asymmetry of intensity can be also seen in the same direction on the X-ray pattern of figure $3 \mathrm{~b}$ ), it is tempting to suggest that $\varphi_{\mathbf{k}}>0$. This corresponds to a contraction of the modulation wave length $2 \pi / 2 \mathbf{k}_{\mathrm{F}}$ in the vicinity of the irradation defects and thus to a local increase of the charge transfer.

\section{Concluding remarks}

We have shown that the strong pinning of CDW's on defects as well as their phase deformation around defects give rise to new diffuse scattering features allowing to determine respectively the phase of the $\mathrm{CDW}$ and its spatial variations in the vicinity of these impurity sites. Analysis of the experimental observations in this theoretical framework surprinsingly shows that molecular substituants act as strong pinning centers in charge transfer salts and that the phase of the CDW is distorded around irradiation defects. Similar features will certainly occur in inorganic quasi-1D conductors. For example asymmetric $2 \mathbf{k}_{\mathrm{F}}$ diffuse lines have been reported in the $\mathrm{V}$ doped blue bronzes [9], but its physical consequences have not been analyzed. These experimental features could also be relevant for other pinning models[12].

\section{REFERENCES}

[1]-KRIVOGLAZ M. A., "Theory of X-ray and Thermal-Neutron Scattering by Real Crystals", Plenum Press, New York (1969).

[2]-GUINIER A., "X-Ray diffraction in Crystals, Imperfect Crystals and Amorphous Bodies", San Francisco: W.H. Freeman and Company (1963).

[3]-BOUVERET Y. and MEGTERT S., J. Phys. France 50 (1989) 1649.

[4]-RAVY S., POUGET J.P. and COMES R., J. Phys. I France 2 (1992) 1173.

[5]-WARREN B.E., "X-ray diffraction", Addison-Wesley Publishing Company (1969).

[6]-FORRO L., ZUPPIROLI L., POUGET J.P. and BECHGAARD K., Phys. Rev. B27 (1983) 7600.

[7]-DEDERICHS P.H., J. Phys. F 3 (1973) 471.

[8]-ZUPPIROLI L., HOUSSEAU N., FORRO L., GUILLOT J.P. and PELISSIER J., Ultramicroscopy 19 (1986) 325.

[9]-GIRAULT S., MOUDDEN A.M., POUGET J.P. and GODARD J.M., Phys. Rev B38 (1988) 7980.

[10]-POUGET J.P., RAVY S. and HENNION B., Phase Transitions 30 (1991) 5.

[11]-FUKUYAMA H. and LEE P.A., Phys. Rev. B17 (1978) 535.

-LEE P.A. and RICE T.M., Phys, Rev. B19 (1979) 3970.

[12]-TÜTTÖ I. and ZAWADOWSKI A., Phys. Rev. B32 (1985) 2449.

-ABE S., J. Phys. Soc. Jpn. 55 (1986) 1987. 